\title{
Large-scale, Lithography-free Production of Transparent Nanostructured Surface for Dual-functional Electrochemical and SERS Sensing
}

Sanger, Kuldeep; Durucan, Onur; Wu, Kaiyu; Thilsted, Anil Haraksingh; Heiskanen, Arto R; Rindzevicius, Tomas; Schmidt, Michael Stenbæk; Zor, Kinga; Boisen, Anja

\section{Published in:}

ACS Sensors

Link to article, DOI:

10.1021/acssensors.7b00783

Publication date:

2017

Document Version

Peer reviewed version

Link back to DTU Orbit

Citation (APA):

Sanger, K., Durucan, O., Wu, K., Thilsted, A. H., Heiskanen, A. R., Rindzevicius, T., Schmidt, M. S., Zor, K., \& Boisen, A. (2017). Large-scale, Lithography-free Production of Transparent Nanostructured Surface for Dualfunctional Electrochemical and SERS Sensing. ACS Sensors, 2(12), 1869-1875.

https://doi.org/10.1021/acssensors.7b00783

\section{General rights}

Copyright and moral rights for the publications made accessible in the public portal are retained by the authors and/or other copyright owners and it is a condition of accessing publications that users recognise and abide by the legal requirements associated with these rights.

- Users may download and print one copy of any publication from the public portal for the purpose of private study or research.

- You may not further distribute the material or use it for any profit-making activity or commercial gain

- You may freely distribute the URL identifying the publication in the public portal 


\title{
Large-Scale, Lithography-Free Production of Transparent Nanostructured Surface for Dual-Functional Electrochemical and SERS Sensing
}

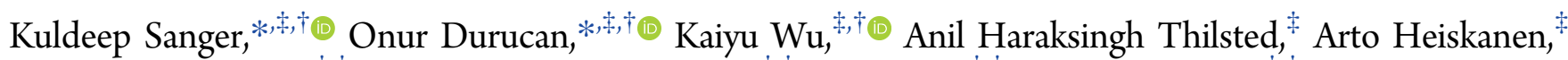 \\ Tomas Rindzevicius, ${ }^{+, \dagger}$ Michael Stenbæk Schmidt, ${ }^{+\dagger}$ Kinga Zór, ${ }^{+, \dagger}$ and Anja Boisen ${ }^{+, \dagger}$ \\ ${ }^{*}$ Department of Micro- and Nanotechnology and ${ }^{\dagger}$ DNRF and Villum Fonden Center for Intelligent Drug Delivery and Sensing Using \\ Microcontainers and Nanomechanics, IDUN, Technical University of Denmark, 2800 Kgs. Lyngby, Denmark
}

\begin{abstract}
In this work, we present a dual-functional sensor that can perform surface-enhanced Raman spectroscopy (SERS) based identification and electrochemical (EC) quantification of analytes in liquid samples. A lithographyfree reactive ion etching process was utilized to obtain nanostructures of high aspect ratios distributed homogeneously on a 4 in. fused silica wafer. The sensor was made up of three-electrode array, obtained by subsequent e-beam evaporation of $\mathrm{Au}$ on nanostructures in selected areas through a shadow mask. The SERS performance was evaluated through surface-averaged enhancement factor (EF), which was $\sim 6.2 \times$ $10^{5}$, and spatial uniformity of EF, which was $\sim 13 \%$ in terms of relative standard deviation. Excellent electrochemical perform-

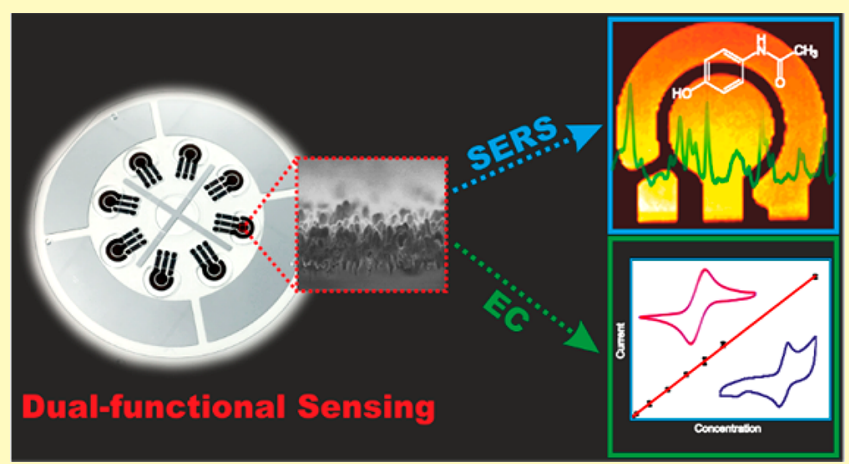
ance and reproducibility were revealed by recording cyclic voltammograms. On nanostructured electrodes, paracetamol (PAR) showed an improved quasi-reversible behavior with decrease in peak potential separation $\left(\Delta E_{\mathrm{p}} \sim 90 \mathrm{mV}\right)$ and higher peak currents $\left(I_{\mathrm{pa}} / I_{\mathrm{pc}} \sim 1\right)$, compared to planar electrodes $\left(\Delta E_{\mathrm{p}} \sim 560 \mathrm{mV}\right)$. The oxidation potential of PAR was also lowered by $\sim 80$ $\mathrm{mV}$ on nanostructured electrodes. To illustrate dual-functional sensing, quantitative evaluation of PAR ranging from $30 \mu \mathrm{M}$ to 3 $\mathrm{mM}$ was realized through EC detection, and the presence of PAR was verified by its SERS fingerprint.
\end{abstract}

KEYWORDS: lithography-free, dual-functional, electrochemical, SERS, paracetamol

$\mathrm{E}$ lectrochemical (EC) detection and Raman spectroscopy have been historically linked since the discovery of surfaceenhanced Raman spectroscopy (SERS), where it was found that nanostructured electrode surfaces, typically achieved via electrochemical roughening, introduced nanoscale hot spots that significantly enhanced the Raman signal. ${ }^{1,2}$

In recent years, SERS has become an analytical tool, allowing detection of analytes at ultralow concentrations by providing their molecular vibrational fingerprints. ${ }^{3-5}$ It has been widely used in biomedical diagnostics, environmental sensing, food safety, and illicit drug control. ${ }^{6-8}$ On the other hand, extensive research has been carried out, using defined nanostructures as electrodes for electrochemical sensing. By using electrodes consisting of nanostructured surfaces, such as nanoporous metallic structures, nanoparticles, ${ }^{9}$ nanotubes, ${ }^{10}$ nanowires, ${ }^{11-13}$ and vertically aligned nanopillars. ${ }^{14,15}$ performance of electrochemical sensing has been improved dramatically, due to the unique electrical, chemical, and catalytic properties, and increased surface areas of the nanostructures. ${ }^{16}$

The combination of SERS with electrochemistry (EC-SERS) has led to research in various areas, including corrosion and surface science, ${ }^{17}$ fuel cells, electrocatalysis, and surface redox reactions, ${ }^{18-20}$ as well as adsorption and reaction of biomolecules. $^{21,22}$ Particularly, EC and SERS have been used as complementary techniques to reveal and explain chemical phenomena, or as a way to improve sensing performance. For example, coupling EC with SERS has been proven beneficial for monitoring electrochemical reactions at ultralow concentrations. In addition, by applying a potential, the adhesion of target analytes to the Au surface can be promoted. This would increase the number of analytes in the electromagnetic hot spots, improving the SERS detection limits. ${ }^{23,24}$ A more advanced technique involves using fluctuation of the applied electrode potential to adsorb and desorb different sets of molecules while monitoring the Raman signatures and thereby avoiding spectral congestion. This is especially relevant with coadsorption of molecules in complex solutions, typically biological solutions. ${ }^{22}$

As application expands by combining EC with SERS, in recent years, focus has been addressed on developing substrates 
A

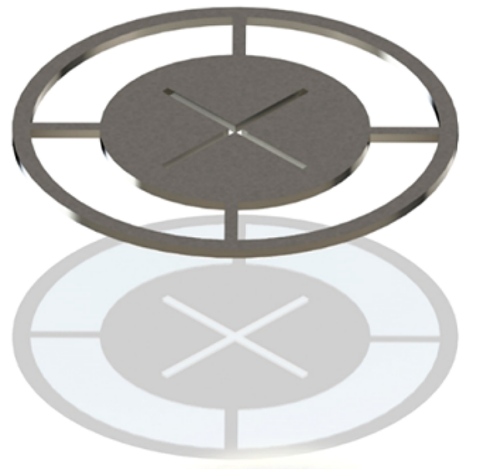

D

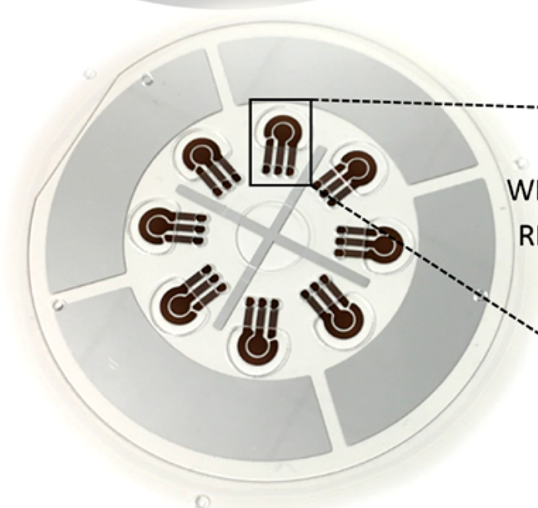

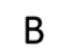

B
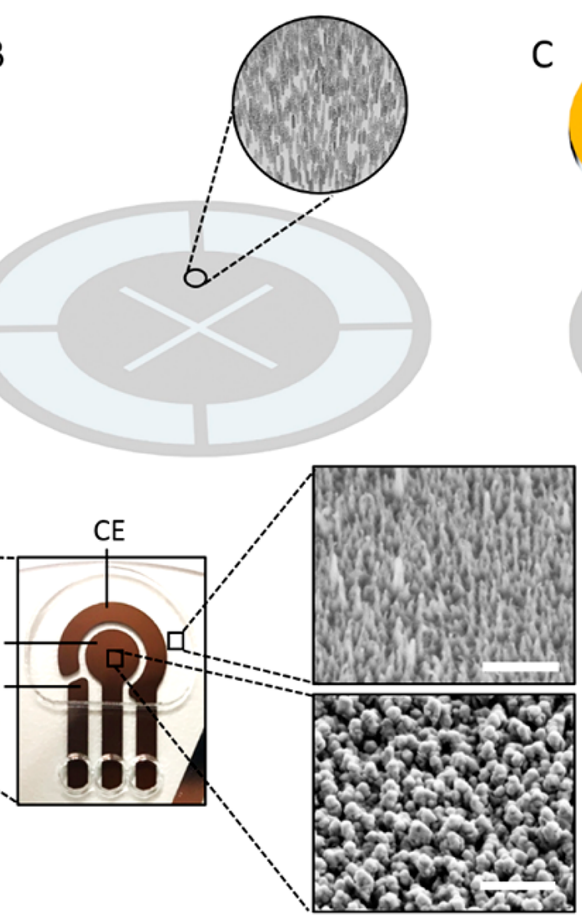

C

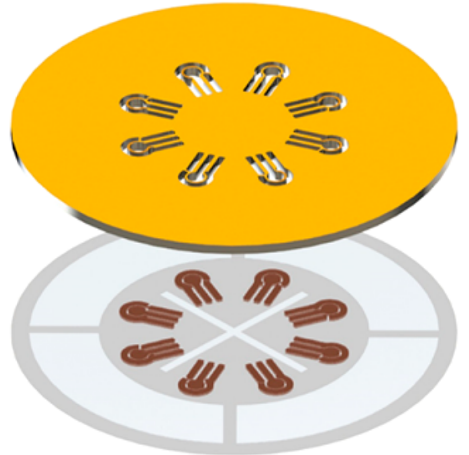

E

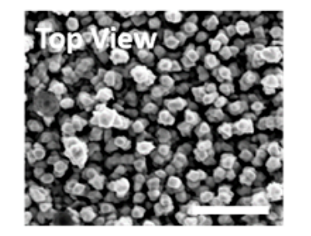

Side View

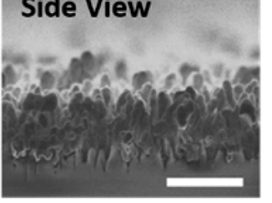

Figure 1. Illustration of the fabrication process of gold capped glass nanopillars. (A) Al layer deposition through a shadowmask, (B) RIE resulting in fused silica nanostructures, (C) electrode patterning using Au deposition through a shadowmask, (D) photograph of whole assembly with inset showing three-electrode arrays, SEM images showing areas of silica nanostructures with (bottom) and without (top) Au deposition, (E) SEM images (top view and side view) of fused silica nanostructures with an evaporated Au layer (All SEM images have scale bar $=1 \mu \mathrm{m}$ ).

that are dual-functional, i.e., integrating EC and SERS sensing on a single chip. In recent studies, Chen et al. reported the deposition of $\mathrm{Ag}$ nanowires on a coffee filter by a simple dipcoating route to develop an enzyme-free sensor for hydrogen peroxide detection. ${ }^{25}$ Bailey et al. reported polystyrene encapsulated $\mathrm{Au}$ electrodes with the electrochemically roughened surface for studying surface chemistry of neurotransmitters. ${ }^{26}$ Furthermore, to study neural stem cell differentiation, Au nanostar arrays were electrochemically deposited on indium tin oxide substrates. ${ }^{27}$ Single cell monitoring was achieved on zinc oxide nanorods grown on planar microelectrodes with $\mathrm{Au}$ electroplating. ${ }^{28}$ For studying biotin-avidin interaction, silicon nanowires were grown on microscope slides by plasma enhanced chemical vapor deposition, and the structures were coated by $\mathrm{Au}$, rendering simultaneously working electrode and SERS probes. ${ }^{29}$ In addition, dual sensing was achieved on commercial $\mathrm{Au}$ electrode and was applied for evaluation of drug intercalation into a DNA duplex. While the molecular identification profiles were provided by SERS, the concentration dependent modification of the DNA by the drug was evaluated by EC detection. ${ }^{30}$

The success of combining EC and SERS relies on the nanostructured enhancing surfaces. Fabrication techniques have typically involved chemical synthesis ${ }^{31}$ and electrodeposition, sometimes in combination with lithographic techniques. ${ }^{32}$ For solid substrates, they are usually patterned by lithography, with a subsequent metallization process. ${ }^{33-35}$ The main drawback of lithographic techniques is that they are time-consuming and costly. Lithography-free reactive ion etching (RIE), on the other hand, has been proven to be a good alternative, since it is much faster and significantly less expensive. ${ }^{36-38}$ For example, using maskless RIE, wafer-scale, high performance SERS substrates consisting of nanopillars that are able to form clusters, can be manufactured. ${ }^{39,40}$ However, the semiconducting nature of silicon has prevented these structures to be used as dual-functional sensors for EC and SERS. Recent developments have shown that lithography-free nanostructuring of insulating and chemically stable materials, such as fused silica (glass), is possible. ${ }^{36,41,42}$ This opens up new possibilities for integrating EC and SERS on a single chip, in a cost-effective manner, which is also reproducible and suitable for mass production.

In this paper, we report an easy to implement, inexpensive, and fast lithography-free process, for manufacturing a dualfunctional EC and SERS sensor system, and present its characterization and application. Dense and homogeneous nanostructures with high aspect ratios were obtained on a 4 in. fused silica wafer using mask-less RIE process. By e-beam evaporation of $\mathrm{Au}$ on these nanostructures through a shadow mask, a circular pattern of eight sets of three-electrode array (comprising working, counter, and reference electrode) was achieved, allowing the system to be used for SERS and EC sensing simultaneously. As a case study, SERS fingerprints were recorded for paracetamol (PAR), followed by its EC quantification.

\section{EXPERIMENTAL SECTION}

Materials. All chemicals and reagents were used as received. Potassium hexacyanoferrate(II) (ferrocyanide), potassium hexacyanoferrate(III) (ferricyanide), phosphate buffered saline (PBS) containing $0.01 \mathrm{M}$ phosphate buffer, $0.0027 \mathrm{M}$ potassium chloride, and $0.137 \mathrm{M}$ sodium chloride ( $\mathrm{pH} 7.4$ ), trans-1,2-bis (4-pyridyl) ethylene (BPE), methanol, and paracetamol were purchased from SigmaAldrich Co. (St. Louis, MO, USA). Absolute ethanol was from 
Kemetyl A/S (Køge, Denmark). All aqueous solutions were prepared in ultrapure water obtained from a Milli- $Q$ water purification system (Millipore Corporation, Billerica, MA, USA). A 4 in. double-sidepolished fused silica wafer with a $525 \mu \mathrm{m}$ thickness (Innergie Technologies, Inc.), 0.6-mm-thick optical grade poly(methyl methacrylate) (PMMA) (Evonik Industries AG, Darmstadt, Germany) and double-sided pressure sensitive adhesive (PSA) tape (140 $\mu \mathrm{m}$ thick, ARcare 90106 from Adhesive Research Ltd., Limerick, Ireland) were used.

Fabrication of Dual-Functional Sensor. The nanostructures were obtained using RIE technique on 4 in. fused silica wafer. Prior to performing RIE process, Al mask (600 nm thick) was deposited through a shadow mask via e-beam evaporator (Wordentec QCL 800 E-beam and sputtering deposition system). The 4 min RIE procedure at an $\mathrm{O}_{2}$ flow of $70 \mathrm{sccm}$, an $\mathrm{SF}_{6}$ flow of $170 \mathrm{sccm}$, a platen power of $110 \mathrm{~W}$, a coil power of $450 \mathrm{~W}$, chuck temperature $5{ }^{\circ} \mathrm{C}$ and a chamber pressure of $75 \mathrm{mTorr}$ was performed using advanced oxide etcher (STS MESC Multiplex ICP). Further, 170-nm-thick Au film in the form of three-electrode array was achieved over the nanostructures using the e-beam evaporator. The morphology of nanostructures was characterized using a scanning electron microscope (Zeiss Supra VP 40 SEM). The planar electrodes were patterned on the fused silica wafer using e-beam evaporation $(17 \mathrm{~nm} \mathrm{Cr}$ adhesion layer/170 nm $\mathrm{Au}$ ) through a shadow mask that was previously used to evaporate $\mathrm{Au}$ on nanostructured surface.

The working area of the electrodes and the wells $(150 \mu \mathrm{L})$ to accommodate the sample over the electrodes were defined by utilizing 0.6 -mm-thick PMMA and 140- $\mu$ m-thick double-sided PSA tape. The pattern to cut PMMA and PSA was designed using the computer aided design (CAD) software SolidWorks (Dassault Systèmes SolidWorks Corporation, Waltham, MA, USA). A computer-controlled Epilog Mini 18 laser cutter equipped with a $30 \mathrm{~W} \mathrm{CO}_{2}$ laser (Epilog Laser, Golden, CO, USA) was used to cut the desired pattern on PMMA, whereas Cameo 3 blade cutter (Silhouette, Inc., Lindon, UT, USA) was utilized to cut the PSA. Finally, the laser-cut PMMA was bonded to the fused silica substrate, incorporating the sensors, using PSA (see Figure 2 for final assembly). Simultaneous electrical connections to all

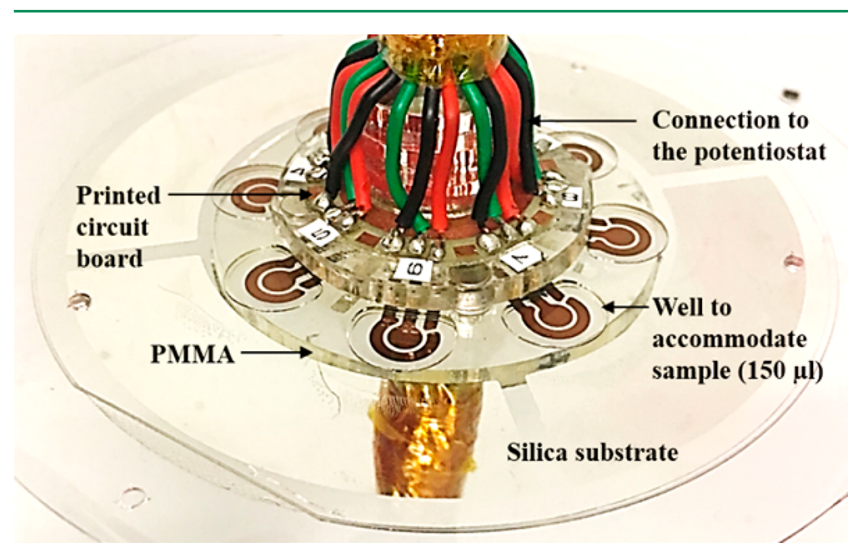

Figure 2. Photograph of the integrated sensors interfaced with a printed circuit board.

eight sets of three-electrode arrays were achieved using custom-made printed circuit board with magnetic clamping, as described in our previous work. ${ }^{43}$

SERS Measurements. SERS measurements were carried out using a Raman microscope (DXRxi Raman Imaging microscope, Thermo Scientific, Waltham, MA, U.S.) equipped with a $780 \mathrm{~nm}$ laser wavelength and a $10 \times$ focusing objective.

Dark-Field Scattering Measurements. A Nikon Ti-U inverted microscope was used with a CF LU Plan Fluor Epi 50× objective, which had a working distance of $1 \mathrm{~mm}$ and a NA of 0.8 . The incident angle was $\sim 50^{\circ}$. The scattered light was collected from the center of the objective and was subsequently guided into a spectrometer (Shamrock Spectrograph SR-303I-A, equipped with an Andor Newton
970 EMCCD). The obtained scattering spectrum was then divided by the reference white light spectrum to obtain the scattering signal from the substrates.

Electrochemical Measurements. All electrochemical measurements were carried out at room temperature using an eight-channel CHI1030A potentiostat (CH Instruments, Inc., Austin, TX, USA) connected simultaneously to eight sets of three-electrode arrays fabricated on $4 \mathrm{in}$. fused-silica wafer. For the electrode characterization, cyclic voltammograms (CVs) were recorded from $-400 \mathrm{mV}$ to 400 $\mathrm{mV}$ vs $\mathrm{Au}$ pseudoreference electrode (RE) in $10 \mathrm{mM}$ ferri/ ferrocyanide using PBS as supporting electrolyte at scan rate $50 \mathrm{mV}$ $\mathrm{s}^{-1}$. Electrochemical fingerprint of PAR in PBS was recorded using CV from $-400 \mathrm{mV}$ to $600 \mathrm{mV}$ vs Au pseudo-RE at scan rate $50 \mathrm{mV} \mathrm{s}^{-1}$. The quantification was performed with batch amperometry by successive additions of PAR at an applied potential of $450 \mathrm{mV}$ vs pseudo-RE in PBS.

\section{RESULTS AND DISCUSSION}

Fabrication Process. The fabrication process of nanostructured surface obtained on fused silica wafer using a lithography-free process (see Experimental Section) is illustrated in Figure 1A,B. A previously described masking technique $^{36}$ was utilized during the RIE process. Accordingly, a seed layer of $\mathrm{Al}$ was used to sputter nanoscale masks onto the fused silica surface. The $\mathrm{Al}$ pattern consists of an outer ring (Figure 1A) to allow electrostatic clamping during the etching procedure. Further, the design also featured a center cross that ensured reduction of the maximum distance of the exposed fused silica wafer to areas with $\mathrm{Al}$ so that sputtering of nanomasks occurs evenly across the wafer. In this way, the arrangement of $\mathrm{Al}$ seed layer promoted fabrication of uniformly distributed dense nanostructures (Figure S1). Subsequent evaporation of $\mathrm{Au}$ on these nanostructures through a shadow mask resulted in formation of isolated metal caps and defined the working, counter, and reference electrodes (Figure 1C). SEM images of the Au capped fused silica nanostructures are shown in Figure 1D,E.

SERS Characterization. SERS performance of the Aucapped nanostructures was evaluated using trans-1,2-bis(4pyridyl)ethylene (BPE) dissolved in ethanol. Figure 3A shows the distribution of the Raman signal intensity, recorded from 5 $\mu \mathrm{L}$ of $1 \mathrm{mM}$ BPE, which was evenly deposited on the sensor surface. In addition, we were able to measure BPE both from the front as well as through the backside of the substrate. As shown in Figure 3C, the measurement through the back of the substrate showed a $37 \%$ lower SERS signal compared to those recorded from the front, with background signal recorded using pure ethanol. These measurements showed a strong enhancement with minimal background signal and the ability to measure from the back of the sensor. Furthermore, we found a homogeneous signal enhancement with a relative standard deviation of $13.4 \%$, as shown in Figure 3C.

The surface-averaged SERS enhancement factor (EF) of the substrate was calculated from the formula:

$$
\mathrm{EF}=\frac{I_{\text {SERS }} / N_{\text {SERS }}}{I_{\mathrm{RS}} / N_{\mathrm{RS}}}
$$

where $I_{\text {SERS }}$ and $I_{\mathrm{RS}}$ were the SERS and the Raman intensity, respectively. $N_{\text {SERS }}$ and $N_{\text {RS }}$ were the numbers of molecules probed in the SERS and the Raman measurements. To establish the value of $I_{\mathrm{RS}}$ and $N_{\mathrm{RS}}$, a $100 \mathrm{mM}$ ethanol solution of BPE was poured into a Petri dish. 1.4 counts $\mathrm{mW}^{-1} \cdot \mathrm{s}^{-1}\left(I_{\mathrm{RS}}\right)$ were measured for the $1200 \mathrm{~cm}^{-1}$ peak under an excitation wavelength of $780 \mathrm{~nm}$. The number of molecules excited $\left(N_{\mathrm{RS}}\right)$ 


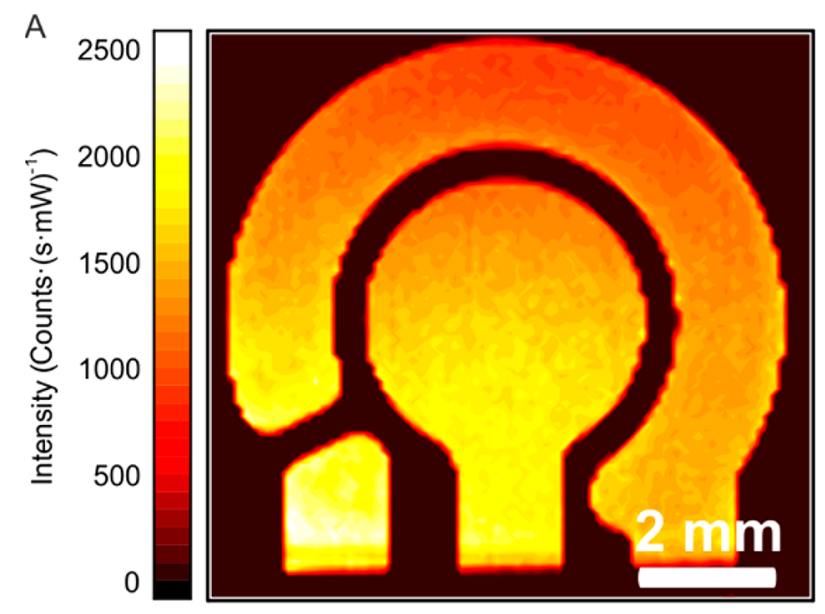

B
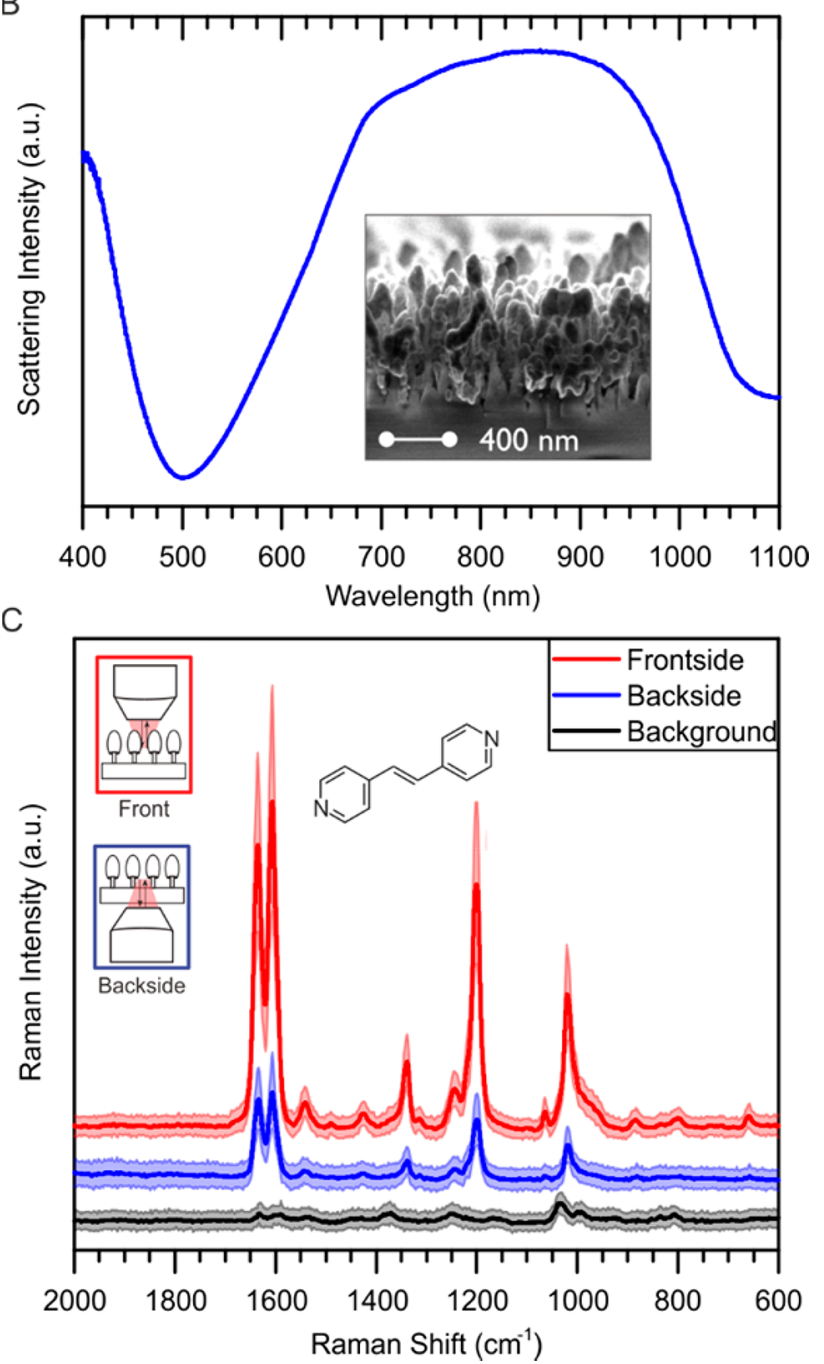

Figure 3. (A) Spatial distribution of the Raman signal at the 1198 $\mathrm{cm}^{-1}$ peak, using $5 \mu \mathrm{L}$ of $1 \mathrm{mM}$ BPE. (B) Dark-field scattering spectrum of the substrate. (C) Raman spectra using a $1 \mu \mathrm{L}$ drop of a $100 \mu \mathrm{M}$ concentration of BPE, measured from the front and backside. The relative standard deviation of the Raman signal was $13.4 \%$. The mean and standard deviation were calculated for wavenumber 1018 $\mathrm{cm}^{-1}$ after background correction. The spectra were collected from 1.3 $\times 1.3 \mathrm{~mm}^{2}$ mapping area with the step size of $100 \mu \mathrm{m}$. Shaded error bars show $90 \%$ confidence interval. was estimated to be $3.60 \times 10^{9}$, using the interaction volume of $60 \mu \mathrm{m}^{3}$. To establish the values for $I_{\text {SERS }}$ and $N_{\text {SERS }}, 1 \mu \mathrm{L}$ of 1 $\mathrm{mM}$ ethanol solution of BPE was pipetted onto the substrate. Hereafter, the substrate was dried in air. The introduced BPE solution forms a quasi-circular pattern on the surface of the substrate with a radius of $\sim 3400 \mu \mathrm{m}$. This leads to a density of $\mathrm{BPE}$ on the substrate to be $1.67 \times 10^{7} / \mu \mathrm{m}^{2}$. Using identical experimental conditions as for $I_{\mathrm{RS}}, \sim 8000 \mathrm{~mW}^{-1} \cdot \mathrm{s}^{-1}$ ( $I_{\text {SERS }}$ ) were measured for the $1200 \mathrm{~cm}^{-1}$ peak. By assuming uniform adsorption of $\mathrm{BPE}$ onto $\mathrm{Au}$ and a laser spot size of $0.8 \mu \mathrm{m}$ in radius, $N_{\text {SERS }}$ is then estimated to be $3.33 \times 10^{7}$. Hence, the calculated surface-averaged EF becomes $6.2 \times 10^{5}$. Note that this EF is a surface-averaged value, obtained by assuming that all the adsorbed molecules contributed equally to the integrated SERS signal, and is thus not the maximum enhancement, which others have attempted to calculate by estimating the number of molecules in the hot spots.

Dark-field scattering spectrum of the substrate is obtained as shown in Figure 3B. A broad peak ranging from $\sim 600 \mathrm{~nm}$ to $\sim 1000 \mathrm{~nm}$ is observed. This is because of the tremendous randomness of the structures, which enables the substrate to support extensive localized plasmon resonance modes. A wide range of excitation wavelengths is thus selectable to perform efficient SERS analysis for different applications. In addition, the dip at $\sim 500 \mathrm{~nm}$ is caused by the interband transition of Au.

EC Characterization. EC performance and reproducibility of the nanostructured electrodes were evaluated by acquiring CVs in $10 \mathrm{mM}$ ferro/ferricyanide dissolved in PBS. The electrodes showed a quasi-reversible electrochemical response with a peak potential separation $\left(\Delta E_{\mathrm{p}}\right)$ of $99 \pm 2 \mathrm{mV}$ at $50 \mathrm{mV}$ $\mathrm{s}^{-1}$ and the ratio between the reduction and oxidation peaks was $1.09 \pm 0.03$ as shown in Figure 4A. The recorded CVs confirm an excellent reproducibility of the electrodes.

According to SEM images shown in Figure 1E, the majority of $\mathrm{Au}$ that defined the electrode area consists of $\mathrm{Au}$ nanoparticles with randomized morphologies. Therefore, the following equation can be established to estimate the 3D surface area of the nanostructured WE, $S_{3 \mathrm{D}}$ :

$$
S_{3 \mathrm{D}}=\overline{S_{\mathrm{NP}}} \times \overline{D_{\mathrm{NP}}} \times S_{2 \mathrm{D}}
$$

where $\overline{S_{\mathrm{NP}}}$ is the average surface area of a $\mathrm{Au}$ nanoparticle, $\overline{D_{\mathrm{NP}}}$ is the average density of the Au nanoparticles, and $S_{2 \mathrm{D}}$ is the two-dimensional surface area of the WE. Approximating each $\mathrm{Au}$ nanoparticle by an ellipsoid with lengths of axes being 100,100 , and $200 \mathrm{~nm}, 54000 \mathrm{~nm}^{2}$ is estimated for $\overline{S_{\mathrm{NP}}}$. From top-view SEM images, $\overline{D_{\mathrm{NP}}}$ is estimated to be $35 \mathrm{um}^{-2}$. As per the electrode design shown in Figure $1 \mathrm{D}, S_{2 \mathrm{D}}$ is $14.92 \mathrm{~mm}^{2}$. These lead to an estimated $S_{3 \mathrm{D}}$ of $28.20 \mathrm{~mm}^{2}$. Thus, the surface area of the nanostructured electrode is increased by a factor of 1.89 relative to the planar electrode. Figure 4B shows a comparison of CVs for nanostructured and planar electrode in PBS. Due to increase in surface area, nanostructured electrode exhibit higher currents for charging double layer capacitance at the electrode/electrolyte interface as compared to the planar electrode. However, the CVs recorded in the presence of redoxactive species did not confirm the significant increase in surface area for nanostructured electrode (Figure 4C). The possible explanation could be that the wetting of the nanostructured electrode was not complete, i.e., the electroactive probe did not have access to the crevices of the nanostructured electrode.

Dual Sensing Detection and Quantification of Paracetamol. PAR was chosen as a model analyte to be identified 


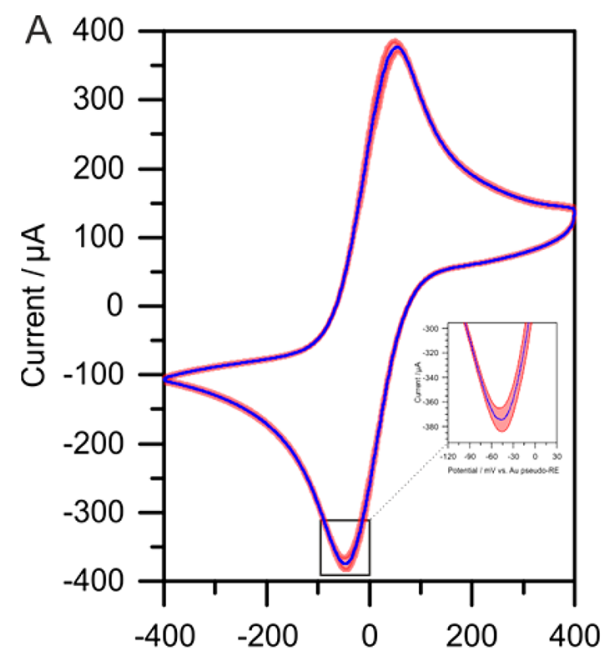

Potential / $\mathrm{mV}$ vs. Au pseudo-RE

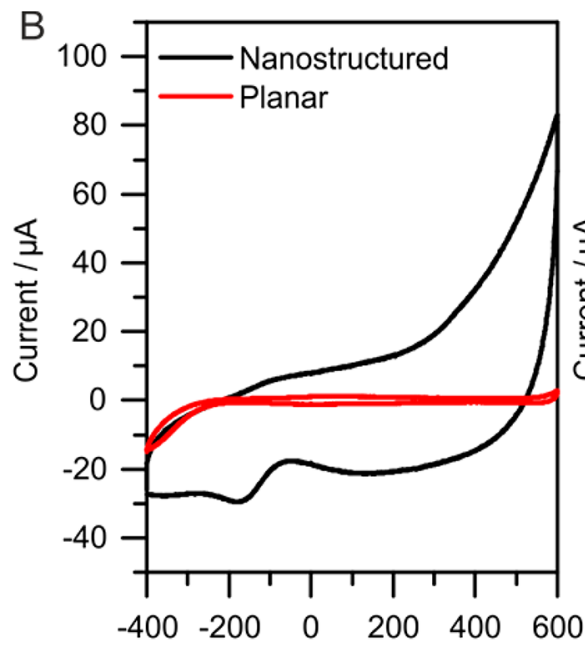

Potential / mV vs. Au pseudo-RE

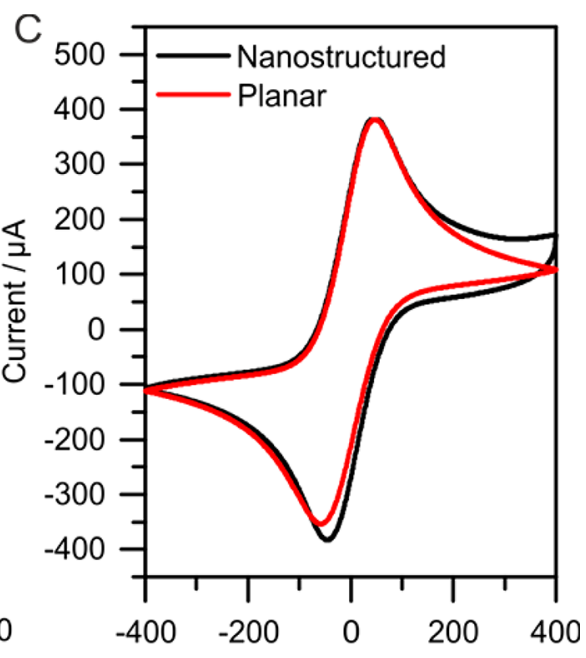

Potential / mV vs. Au pseudo-RE

Figure 4. $\mathrm{CVs}$ for (A) 16 electrodes patterned on two different substrates (each carrying 8 electrodes) acquired in $10 \mathrm{mM}$ ferri/ferrocyanide using PBS as supporting electrolyte, red shaded area shows the standard deviation among the electrodes. (B) and (C) planar Au electrode vs nanostructured gold electrode in PBS and in $10 \mathrm{mM}$ ferri/ferrocyanide using PBS as supporting electrolyte, respectively. (scan rate $50 \mathrm{mVs}{ }^{-1}$.)
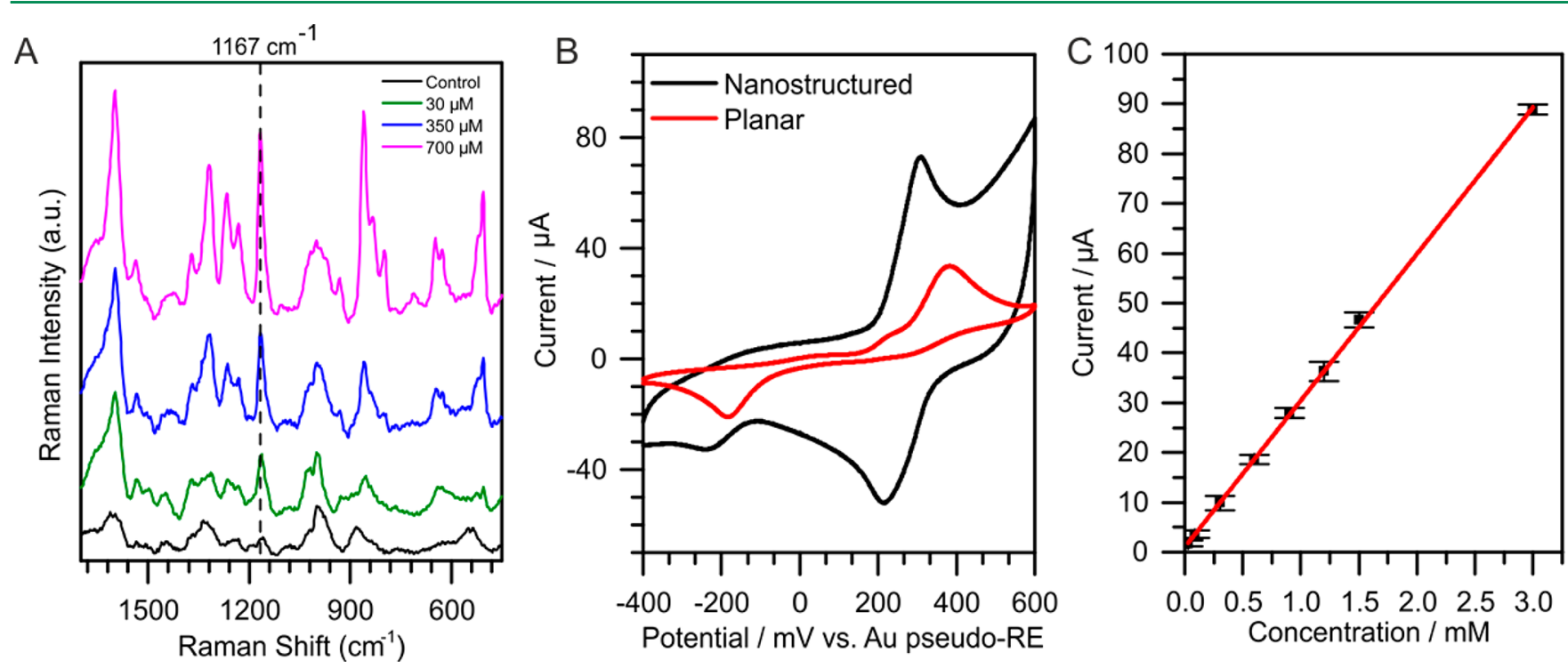

Figure 5. (A) SERS measurement of paracetamol in PBS showing clear peaks for different concentrations of paracetamol. (B) CV of $1 \mathrm{mM}$ PAR on planar and nanostructured electrodes. (C) Calibration curve of paracetamol obtained by amperometry at an applied potential of $450 \mathrm{mV}$ in PBS vs Au pseudo-RE.

and quantified using the developed dual-functional sensor. As shown in Figure 5A, the presence of PAR, based on its Raman fingerprint, was confirmed with SERS-sensing at different concentrations. For SERS detection, in order to reduce interferences caused by salts present in the PBS and to increase the wettability of the sensing surface, the samples were diluted with $10 \%$ methanol. In comparison with the control measurement, distinct fingerprint peaks were observed for paracetamol (e.g., vibrational mode at $1167 \mathrm{~cm}^{-1}$ ).

The improved electrochemical behavior of PAR on surface modified glassy carbon electrodes have been already shown in the literature. ${ }^{44-46}$ However, the presented nanostructured electrode exhibited an excellent electrocatalytic activity to PAR without any surface modification as compared to the planar electrode with similar geometry. In the case of planar electrode, the PAR was oxidized at $380 \mathrm{mV}$, whereas the oxidation potential was lowered by $80 \mathrm{mV}$ for a nanostructured electrode with higher peak currents $\left(I_{\mathrm{pa}} / I_{\mathrm{pc}} \sim 1\right)$ (Figure 5B). This can explain an efficient catalytic role of the Au-coated nanostructured surface that provides enough reaction sites for the enhancement of the current response toward PAR. For $1 \mathrm{mM}$ $\mathrm{PAR}$, oxidation and reduction peak currents on nanostructured electrodes were $40 \mu \mathrm{A} \pm 2$ and $40 \mu \mathrm{A} \pm 2$, and on planar electrodes were $22 \mu \mathrm{A} \pm 2$ and $15 \mu \mathrm{A} \pm 1$, respectively. In addition, PAR showed an improved quasi-reversible behavior on the nanostructured electrode with peak potential separation $\left(\Delta E_{\mathrm{p}}\right)$ of $90 \mathrm{mV}$ as compared to $560 \mathrm{mV}$ for the planar electrode. The decrease in the $\Delta E_{\mathrm{p}}$ value of the nanostructured electrode confirms the effective electron transfer ability of the Au-coated nanostructured surface. The CV was also acquired in $100 \mathrm{mM}$ PBS with or without PAR to confirm that the oxidation and reduction peaks solely belongs to the PAR (Figure S2). As shown in Figure 5C, the linear range, from 30 $\mu \mathrm{M}$ up to $3.0 \mathrm{mM}$, was obtained when quantifying PAR on the 
nanostructured electrodes using amperometry at an applied potential of $450 \mathrm{mV}$. In addition, attributing to the slightly higher surface area, nanostructured electrodes showed improved sensitivity as compared to the planar electrodes during quantification (Figure S3).

\section{CONCLUSION}

A dual-functional sensor for EC and SERS-sensing consisting of nanostructured three-electrode array was developed. The sensor was successfully applied for quantitative detection of PAR, during which EC and SERS served, respectively, for quantification and identification, complementing each other. The developed lithography-free fabrication process is reproducible, high-throughput, and inexpensive, and is thus suitable for commercialization. The high optical transparency of the substrate allows excitation and collection of SERS spectra from the backside of the sensors. Thereby, detecting analytes in complex samples containing, e.g., cells, becomes possible. In addition, the sensors patterned on a circular wafer would be suitable for integration into centrifugal microfluidic platforms, where a certain part of the assay, such as sample filtration and analyte concentration, can be performed before sensing.

\section{AUTHOR INFORMATION}

\section{Corresponding Authors}

*E-mail: sakuld@nanotech.dtu.dk. Phone: +45 71421426.

*E-mail: onurd@nanotech.dtu.dk. Phone: +45 71515189.

\section{ORCID}

Kuldeep Sanger: 0000-0003-0094-4063

Onur Durucan: 0000-0003-1342-3611

Kaiyu Wu: 0000-0002-5666-8419

Notes

The authors declare no competing financial interest.

\section{ACKNOWLEDGMENTS}

This work was financially supported by the European Research Council under the European Union's Seventh Framework Program (FP7/2007-2013) Grant no. 320535-HERMES and the IDUN Center of Excellence (grant no. DNRF122) funded by the Danish National Research Foundation and the Villum Foundation (Grant No. 9301).

\section{REFERENCES}

(1) Fleischmann, M.; Hendra, P. J.; McQuillan, A. J. Raman Spectra of Pyridine Adsorbed at a Silver Electrode. Chem. Phys. Lett. 1974, 26, $163-166$.

(2) Jeanmaire, D. L.; Van Duyne, R. P. Surface Raman Spectroelectrochemistry. J. Electroanal. Chem. Interfacial Electrochem. 1977, 84, 1-20.

(3) Hakonen, A.; Svedendahl, M.; Ogier, R.; Yang, Z.-J.; Lodewijks, K.; Verre, R.; Shegai, T.; Andersson, P. O.; Käll, M. Dimer-on-Mirror
SERS Substrates with Attogram Sensitivity Fabricated by Colloidal Lithography. Nanoscale 2015, 7, 9405-9410.

(4) Graham, D.; van Duyne, R.; Ren, B. Surface-Enhanced Raman Scattering. Analyst 2016, 141, 4995-4995.

(5) Schlücker, S. Surface-Enhanced Raman Spectroscopy: Concepts and Chemical Applications. Angew. Chem., Int. Ed. 2014, 53, 47564795.

(6) McNay, G.; Eustace, D.; Smith, W. E.; Faulds, K.; Graham, D. Surface-Enhanced Raman Scattering (SERS) and Surface-Enhanced Resonance Raman Scattering (SERRS): A Review of Applications. Appl. Spectrosc. 2011, 65, 825-837.

(7) Sharma, B.; Frontiera, R. R.; Henry, A.; Ringe, E.; Van Duyne, R. P. SERS: Materials, Applications, and the Future. Mater. Today 2012, $15,16-25$.

(8) Hering, K.; Cialla, D.; Ackermann, K.; Dörfer, T.; Möller, R.; Schneidewind, H.; Mattheis, R.; Fritzsche, W.; Rösch, P.; Popp, J. SERS: A Versatile Tool in Chemical and Biochemical Diagnostics. Anal. Bioanal. Chem. 2008, 390, 113-124.

(9) Yu, A.; Liang, Z.; Cho, J.; Caruso, F. Nanostructured Electrochemical Sensor Based on Dense Gold Nanoparticle Films. Nano Lett. 2003, 3, 1203-1207.

(10) Ahammad, A. J. S.; Lee, J.; Rahman, M. A. Electrochemical Sensors Based on Carbon Nanotubes. Sensors 2009, 9, 2289-2319.

(11) Tang, J.; Kong, B.; Wang, Y.; Xu, M.; Wang, Y.; Wu, H.; Zheng, G. Photoelectrochemical Detection of Glutathione by IrO2-HeminTiO2 Nanowire Arrays. Nano Lett. 2013, 13, 5350-5354.

(12) Tang, J.; Wang, Y.; Li, J.; Da, P.; Geng, J.; Zheng, G. Sensitive Enzymatic Glucose Detection by $\mathrm{TiO} 2$ Nanowire Photoelectrochemical Biosensors. J. Mater. Chem. A 2014, 2, 6153-6157.

(13) Dhahi, T. H. S.; Bin Hashim, U. D. A.; Ahmed, N. M.; Mat Taib, A. A Review on the Electrochemical Sensors and Biosensors Composed of Nanogaps as Sensing Material. Journal of Optoelectronics and Advanced Materials 2010, 12, 1857-1862.

(14) Shin, C.; Shin, W.; Hong, H. G. Electrochemical Fabrication and Electrocatalytic Characteristics Studies of Gold Nanopillar Array Electrode (AuNPE) for Development of a Novel Electrochemical Sensor. Electrochim. Acta 2007, 53, 720-728.

(15) Anandan, V.; Rao, Y. L.; Zhang, G. Nanopillar Array Structures for Enhancing Biosensing Performance. Int. J. Nanomedicine 2006, 1, 73-79.

(16) Zhang, J.; Li, C. M. Nanoporous Metals: Fabrication Strategies and Advanced Electrochemical Applications in Catalysis, Sensing and Energy Systems. Chem. Soc. Rev. 2012, 41, 7016.

(17) Cai, W. B.; Ren, B.; Li, X. Q.; She, C. X.; Liu, F. M.; Cai, X. W.; Tian, Z. Q. Investigation of Surface-Enhanced Raman Scattering from Platinum Electrodes Using a Confocal Raman Microscope: Dependence of Surface Roughening Pretreatment. Surf. Sci. 1998, 406, 9-22.

(18) Zong, C.; Chen, C. J.; Zhang, M.; Wu, D. Y.; Ren, B. Transient Electrochemical Surface-Enhanced Raman Spectroscopy: A Millisecond Time-Resolved Study of an Electrochemical Redox Process. J. Am. Chem. Soc. 2015, 137, 11768-11774.

(19) Wu, D.-Y.; Liu, X.-M.; Duan, S.; Xu, X.; Ren, B.; Lin, S.-H.; Tian, Z.-Q. Chemical Enhancement Effects in SERS Spectra: A Quantum Chemical Study of Pyridine Interacting with Copper, Silver, Gold and Platinum Metals. J. Phys. Chem. C 2008, 112, 4195-4204.

(20) Park, S.; Yang, P.; Corredor, P.; Weaver, M. J. Transition MetalCoated Nanoparticle Films: Vibrational Characterization with SurfaceEnhanced Raman Scattering. J. Am. Chem. Soc. 2002, 124, 2428-2429.

(21) Murgida, D. H.; Hildebrandt, P. Disentangling Interfacial Redox Processes of Proteins by SERR Spectroscopy. Chem. Soc. Rev. 2008, 37, 937.

(22) Cortés, E.; Etchegoin, P. G.; Le Ru, E. C.; Fainstein, A.; Vela, M. E.; Salvarezza, R. C. Electrochemical Modulation for Signal Discrimination in Surface Enhanced Raman Scattering (SERS). Anal. Chem. 2010, 82, 6919-6925.

(23) Tian, Z.; Ren, B. Adsorption and Reaction at Electrochemical Interfaces as Probed by Surface-Enhanced Raman Spectroscopy. Annu. Rev. Phys. Chem. 2004, 55, 197-229. 
(24) Dos Santos, D. P.; Andrade, G. F. S.; Temperini, M. L. A.; Brolo, A. G. Electrochemical Control of the Time-Dependent Intensity Fluctuations in Surface-Enhanced Raman Scattering (SERS). J. Phys. Chem. C 2009, 113, 17737-17744.

(25) Chen, Y. C.; Hsu, J. H.; Lin, Y. G.; Hsu, Y. K. Silver Nanowires on Coffee Filter as Dual-Sensing Functionality for Efficient and LowCost SERS Substrate and Electrochemical Detection. Sens. Actuators, B 2017, 245, 189-195.

(26) Bailey, M. R.; Martin, R. S.; Schultz, Z. D. Role of Surface Adsorption in the Surface-Enhanced Raman Scattering and Electrochemical Detection of Neurotransmitters. J. Phys. Chem. C 2016, 120, 20624-20633.

(27) El-Said, W. A.; Kim, S. U.; Choi, J.-W. Monitoring in Vitro Neural Stem Cell Differentiation Based on Surface-Enhanced Raman Spectroscopy Using a Gold Nanostar Array. J. Mater. Chem. C 2015, 3, $3848-3859$.

(28) Zong, X.; Zhu, R.; Guo, X. Nanostructured Gold Microelectrodes for SERS and EIS Measurements by Incorporating $\mathrm{ZnO}$ Nanorod Growth with Electroplating. Sci. Rep. 2015, 5, 16454.

(29) Convertino, A.; Mussi, V.; Maiolo, L. Disordered Array of Au Covered Silicon Nanowires for SERS Biosensing Combined with Electrochemical Detection. Sci. Rep. 2016, 6, 25099.

(30) Ilkhani, H.; Hughes, T.; Li, J.; Zhong, C. J.; Hepel, M. Nanostructured SERS-Electrochemical Biosensors for Testing of Anticancer Drug Interactions with DNA. Biosens. Bioelectron. 2016, 80, 257-264.

(31) Leopold, N.; Lendl, B. A New Method for Fast Preparation of Highly Surface-Enhanced Raman Scattering (SERS) Active Silver Colloids at Room Temperature by Reduction of Silver Nitrate with Hydroxylamine Hydrochloride. J. Phys. Chem. B 2003, 107, 57235727.

(32) Soleymani, L.; Fang, Z.; Sun, X.; Yang, H.; Taft, B. J.; Sargent, E. H.; Kelley, S. O. Nanostructuring of Patterned Microelectrodes to Enhance the Sensitivity of Electrochemical Nucleic Acids Detection. Angew. Chem., Int. Ed. 2009, 48, 8457-8460.

(33) Hakonen, A.; Wang, F.; Andersson, P. O.; Wingfors, H.; Rindzevicius, T.; Schmidt, M. S.; Soma, V. R.; Xu, S.; Li, Y.; Boisen, A.; et al. Hand-Held Femtogram Detection of Hazardous Picric Acid with Hydrophobic Ag Nanopillar SERS Substrates and Mechanism of Elasto-Capillarity. ACS Sensors 2017, 2, 198-202.

(34) Hicks, E. M.; Zhang, X.; Zou, S.; Lyandres, O.; Spears, K. G.; Schatz, G. C.; Van Duyne, R. P. Plasmonic Properties of Film over Nanowell Surfaces Fabricated by Nanosphere Lithography. J. Phys. Chem. B 2005, 109, 22351-22358.

(35) Liu, S.; Xu, Z.; Sun, T.; Zhao, W.; Wu, X.; Ma, Z.; Xu, H.; He, J.; Chen, C. Large-Scale Fabrication of polymer/Ag Core-Shell Nanorod Array as Flexible SERS Substrate by Combining Direct Nanoimprint and Electroless Deposition. Appl. Phys. A: Mater. Sci. Process. 2014, 115, 979-984.

(36) Thilsted, A. H.; Pan, J. Y.; Wu, K.; Zór, K.; Rindzevicius, T.; Schmidt, M. S.; Boisen, A. Lithography-Free Fabrication of Silica Nanocylinders with Suspended Gold Nanorings for LSPR-Based Sensing. Small 2016, 12, 6745-6752.

(37) Zaidi, S. H.; Ruby, D. S.; Gee, J. M. Characterization of Random Reactive Ion Etched-Textured Silicon Solar Cells. IEEE Trans. Electron Devices 2001, 48, 1200-1206.

(38) Gharghi, M.; Sivoththaman, S. Formation of Nanoscale Columnar Structures in Silicon by a Maskless Reactive Ion Etching Process. J. Vac. Sci. Technol., A 2006, 24, 723-727.

(39) Schmidt, M. S.; Hübner, J.; Boisen, A. Large Area Fabrication of Leaning Silicon Nanopillars for Surface Enhanced Raman Spectroscopy. Adv. Mater. 2012, 24, OP11-OP18.

(40) Wu, K.; Rindzevicius, T.; Stenbæk Schmidt, M.; Mogensen, K. B.; Hakonen, A.; Boisen, A. Wafer-Scale Leaning Silver Nanopillars for Molecular Detection at Ultra-Low Concentrations. J. Phys. Chem. C 2015, 119, 2053-2062.

(41) Hein, E.; Fox, D.; Fouckhardt, H. Glass Surface Modification by Lithography-Free Reactive Ion Etching in an Ar/CF4-Plasma for
Controlled Diffuse Optical Scattering. Surf. Coat. Technol. 2011, 205, S419-S424.

(42) Zeze, D. A.; Cox, D. C.; Weiss, B. L.; Silva, S. R. P. LithographyFree High Aspect Ratio Submicron Quartz Columns by Reactive Ion Etching. Appl. Phys. Lett. 2004, 84, 1362-1364.

(43) Sanger, K.; Zór, K.; Heiskanen, A; Amato, L.; Toftgaard Nielsen, A.; Bille Jendresen, C.; Boisen, A. Lab-on-a-Disc Platform for Screening of Genetically Modified E. Coli Cells via Cell-Free Electrochemical Detection of P-Coumaric Acid. Sens. Actuators, B 2017, 253, 999-1005.

(44) Gowda, J. I.; Gunjiganvi, D. G.; Sunagar, N. B.; Bhat, M. N.; Nandibewoor, S. T. MWCNT-CTAB Modified Glassy Carbon Electrode as a Sensor for the Determination of Paracetamol. RSC Adv. 2015, 5, 49045-49053.

(45) Devaraj, M.; Deivasigamani, R. K.; Jayadevan, S. Controlled Growth and Molecular Self-Assembly of $\mathrm{Au}$ Nanoparticles to $\mathrm{Au}$ Nanochains: Application towards Enhancement for the Electrochemical Determination of Paracetamol. Anal. Methods 2013, 5, 3503.

(46) Kang, X.; Wang, J.; Wu, H.; Liu, J.; Aksay, I. A.; Lin, Y. A Graphene-Based Electrochemical Sensor for Sensitive Detection of Paracetamol. Talanta 2010, 81, 754-759. 